\title{
Evaluación de la Escala EPInfant durante una prueba de ejercicio incremental en cinta rodante
}

\author{
IVÁN RODRÍGUEZ-NÚÑEZ*,**,****,*****, ROMINA JEREZ O.**, AVELINA MORA G.**, \\ DANIELA MELLADO M.**, MICHELLE LOUVAES GARCÍA****, DARWIN GATICA S.**,***, \\ HÉCTOR TORRES G.** y CARLOS MANTEROLA D.************
}

\section{Evaluation of the EPInfant scale during an incremental exercise test in treadmill}

Introduction: The scales of perceived exertion (PE) quantify the perceptual changes induced by physiological stress during exercise. Whereas the adult scales cannot be used in children, it was built the EPInfant scale for Spanish speaking children. The aim of this study is to determine the validity of the criteria for an exercise test on a treadmill. Methods: Cross-sectional study, in which the criterion validity was determined by linear regression between the EP and variable intensity exercise (heart rate and workload) during incremental exercise test in treadmill (Naughton's test). Results: At study entered 26 healthy children (13 males) with a mean age of $13.6 \pm 0.13$ years-old. Both the PE and heart rate (HR) increased significantly at every level of intensity during the Naughton' test. It was observed a strong linear correlation between the PE and HR, as well as between PE and workload (speed and slope of the treadmill). All regression models were highly significant $(p<0.0001)$. Conclusion: EPInfant scale has an acceptable criterion-related validity to measure PE during an incremental exercise test on a treadmill in the studied sample of children. Future studies are needed to determine other properties of measurement in this type of exercise.

Key words: Adolescent; exercise test; heart rate; perception; physical exertion.

\section{Resumen}

Introducción: Las escalas de esfuerzo percibido (EP) permiten cuantificar los cambios perceptuales inducidos por el estrés fisiológico durante el ejercicio. Considerando que las escalas de EP para adultos no pueden ser utilizados en niños, se construyó la escala EPInfant. El objetivo de este estudio fue determinar su validez de criterio durante una prueba de ejercicio en cinta rodante. Métodos: Estudio de corte transversal. Se determinó validez de criterio concurrente aplicando regresión lineal entre EP y variables de intensidad de ejercicio (frecuencia cardiaca $(F C)$ y carga de trabajo), durante la prueba de ejercicio incremental en cinta rodante (test de Naughton), en población infantil sana de la ciudad de Concepción Chile. Resultados: Se estudiaron 26 sujetos (13 varones), con una media de edad de 13,6 \pm 0,13. Tanto la FC como el EP incrementaron significativamente en cada nivel de intensidad durante el test de Naughton. Se verificó correlación lineal entre el EP y FC; así como también, entre EP y carga de trabajo (velocidad e inclinación de la trotadora). Todos los modelos de regresión fueron significativos ( $p<0,0001)$. Conclusión: La escala EPInfant posee una adecuada validez de criterio concurrente para medir EP durante el test de Naughton en la muestra estudiada. Se requieren estudios adicionales para determinar otras propiedades de medición en esta modalidad de ejercicio.

Palabras clave: Prueba de ejercicio; percepción; frecuencia cardíaca; esfuerzo físico.

Financiamiento: Este estudio fue financiado por la Escuela de Kinesiología de la Universidad Santo Tomás, Concepción, Chile.

* Laboratorio de Biología del Ejercicio, Escuela de Kinesiología, Facultad de Ciencias de la Salud, Universidad San Sebastián, Concepción, Chile.

** Escuela de Kinesiología, Facultad de Salud, Universidad Santo Tomás, Concepción, Chile.

*** Laboratorio de Sueño, Clínica Universitaria, Hualpén, Chile.

**** Laboratório de Fisiologia Cardiovascular e Respiratória, Departamento de Fisiologia, Escola Paulista de Medicina, Universidade Federal de São Paulo (EPM-UNIFESP), Brasil.

***** Departamento de Cirugía y CEMyQ, Universidad de La Frontera, Temuco, Chile.

$* * * * * *$ Centro de Investigación en Biociencias, Universidad Autónoma de Chile, Temuco, Chile. 


\section{Introducción}

Las escalas de esfuerzo percibido (EP) fueron desarrolladas para cuantificar los cambios perceptuales inducidos por el estrés fisiológico durante el ejercicio ${ }^{1}$. El principio de su utilización se basa en la interdependencia existente entre la percepción y la intensidad de ejercicio, lo cual, es demostrado mediante la correlación entre el nivel de EP y parámetros de estrés fisiológicos como frecuencia cardíaca (FC) o el consumo de oxígeno $\left(\mathrm{VO}_{2}\right)^{2}$.

Considerando que el proceso perceptivo depende del desarrollo cognitivo de las personas, los instrumentos de medición de EP diseñados para adultos no pueden ser utilizados en niños; lo que, ha sido ampliamente demostrado en la escasa literatura disponible ${ }^{3}$. Recientemente Rodríguez et al., mediante una revisión sistemática con metaanálisis, confirmaron que la medición del EP en menores de 18 años presenta un alto nivel de validez de criterio. Adicionalmente establecieron, que las propiedades psicométricas de la medición del EP en niños depende de su desarrollo cognitivo y de las características de la escala utilizada; al determinar que la medición del EP a través de instrumentos adaptados a población infantil tendría mayor correlación con algunas variables fisiológicas como la frecuencia cardíaca (FC) y el consumo de oxígeno $\left(\mathrm{VO}_{2}\right)$, respecto de las escalas para adultos ${ }^{4}$.

En este contexto, fue recientemente desarrollada la escala EPInfant (Esfuerzo Percibido Infantil); la que fue construida aplicando una metodología que procuró disminuir los potenciales sesgos inherentes a este tipo de estudios; que pudiesen afectar las propiedades psicométricas de este instrumento $^{5}$. Esta escala posee 11 descriptores numéricos ( 0 a 10), 5 descriptores verbales ubicados cada dos niveles de intensidad; y un conjunto de ilustraciones que representan a un niño corriendo a intensidades crecientes a lo largo de una escala de barras de altura incremental, siguiendo una pendiente exponencial de izquierda a derecha ${ }^{6}$.

La escala EPInfant ha sido validada en niños y adolescentes chilenos durante ejercicios desarrollados en escalón; y se constató que posee propiedades psicométricas aceptables para su utilización en sujetos de este grupo de edad ${ }^{7}$. Considerando que la modalidad de ejercicio pudiese ser un factor confundente en la validez de este instrumento; resulta fundamental realizar estudios de validez para cada tipo de ejercicio y modalidad de entrenamiento ${ }^{8}$. Hasta ahora, la escala EPInfant no había sido evaluada durante una prueba estandarizada en trotadora; por ende, el objetivo de este estudio fue determinar la validez de criterio concurrente de la escala EPInfant durante una prueba de ejercicio incremental en cinta rodante en una muestra de niños chilenos.

\section{Material y Métodos}

\section{Diseño}

Estudio de corte transversal, en el cual, la medición del EP fue realizado mediante el paradigma de estimación perceptual durante una prueba de ejercicio incremental en cinta rodante (test de Naughton).

Muestra de estudio: A través de un muestreo no probabilístico por conveniencia fueron seleccionados menores de 18 años, de ambos géneros, provenientes de un colegio público de la ciudad de Concepción, Chile. Antes del inicio del protocolo de estudio, cada uno de los sujetos fue sometido a un examen físico torácico y se completó una lista de chequeo destinada a descartar la presencia de alguna enfermedad cardiorrespiratoria concomitante. Se excluyeron sujetos con déficit cognitivo, obesos y aquellos con patología neurológica o músculo-esquelética. Los padres de los sujetos firmaron consentimiento informado antes de ingresar al estudio. La ejecución del estudio fue autorizada por el comité de ética institucional.

\section{Protocolo}

El estudio fue realizado en el Laboratorio de Fisiología del Ejercicio de la Universidad Santo Tomás (Concepción, Chile). Antes de la prueba de ejercicio, cada sujeto fue familiarizado con la escala EPInfant de acuerdo a las instrucciones publicadas por nuestro grupo ${ }^{5}$. Adicionalmente fueron registrados el peso y la estatura, mediante una balanza análoga y cinta métrica; el porcentaje de grasa, mediante medición de pliegues (bicipital, tricipital, suprailíaco y subescapular); la FC de reposo, mediante un monitor cardiorrespiratorio Polar ${ }^{\circledR}$; y la presión arterial (sistólica y diastólica), mediante un monitor Omron HEM-705 CP.

La prueba de ejercicio utilizada (test de Naughton), consiste en correr sobre una cinta rodante a niveles incrementales de velocidad e inclinación. El test posee 9 niveles de intensidad que van desde $2,6 \mathrm{~km} / \mathrm{h}$ e inclinación $0 \%$, hasta velocidad $7,7 \mathrm{~km} / \mathrm{h}$ e inclinación de $15 \%$; y cada nivel tiene una duración de $2 \mathrm{~min}$. Durante la prueba, la FC y el EP fueron registrados por dos evaluadores independientes durante los últimos $15 \mathrm{~s}$ de cada nivel de intensidad.

Análisis estadístico: con el software estadístico MedCalc (MedCalc Statistical Software versión 
15,8 (MedCalc Software bvba, Ostend, Belgium; https://www.medcalc.org; 2015); se realizó el análisis exploratorio de los datos con evaluación de normalidad mediante el test de Shapiro Wilk. Una vez verificada la distribución normal de los datos se aplicó estadística descriptiva con cálculo de promedio y error estándar (EE). Para determinar la significancia en las diferencias de la FC y EP entre cada nivel de intensidad se aplicó ANOVA de medidas repetidas. Con el fin de evaluar la diferencia de las variables cuantitativas entre géneros, se utilizó t-student para muestras independientes. La validez de criterio concurrente fue determinada de acuerdo a la metodología empleada en estudios previos ${ }^{7}$, donde se considera el análisis de regresión lineal entre el EP y los parámetros de referencia $(\mathrm{FC}$, velocidad de carrera y porcentaje de inclinación de la cinta rodante). Los componentes de la ecuación de regresión son expresados en valor absoluto y $95 \%$ de intervalo de confianza (95\% IC). Finalmente, de acuerdo al número de sujetos y los coeficientes de correlación observados en este reporte, el riesgo de error alfa calculado fue de $1 \%$ y la potencia estadística de $90 \%$

\section{Resultados}

\section{Características generales de la muestra}

$\mathrm{Al}$ estudio ingresaron 26 sujetos (13 varones) sanos con una media de edad de 13,6 $\pm 0,13$ (1315 años); un peso de $52,5 \pm 1,3 \mathrm{~kg}$; una estatura de 1,5 $\pm 0,0 \mathrm{~m}$. No existió diferencia estadística entre los géneros en cuanto a edad, estatura y peso. Sin embargo, el IMC en los varones fue significativamente menor que en las niñas $(20,1 \pm$
$0,5 \mathrm{~kg} / \mathrm{m}^{2}$ en varones y $21,7 \pm 0,5 \mathrm{~kg} / \mathrm{m}^{2}$ en niñas; $\mathrm{p}=0,0421)$. En adición a lo anterior, el porcentaje de grasa fue significativamente mayor en las niñas, quienes presentaron un $27,7 \pm 0,9 \%$ y los varones $21,1 \pm 1,4 \%(\mathrm{p}=0,0008)$.

En relación a los parámetros cardiorrespiratorios de reposo, la frecuencia respiratoria fue de $18,4 \pm 0,5$ ciclos/min en la muestra total, no verificándose diferencias entre géneros. Por su parte, la frecuencia cardíaca en los varones fue de 70,1 $\pm 2,9$ latidos/min y en las niñas $81,7 \pm 1,4$ latidos/min $(\mathrm{p}=0,0024)$. Con respecto a la presión arterial (PA), la PA sistólica, PA diastólica y PA media fueron menores en los varones respecto a las niñas (varones 112,0 $\pm 2,0 \mathrm{mmHg}, 72,0 \pm$ $1,5 \mathrm{mmHg}$ y $85,3 \pm 1,4 \mathrm{mmHg}$; niñas $121,5 \pm 2,7$ $\mathrm{mmHg}, 79,6 \pm 2,9 \mathrm{mmHg}$ y $93,4 \pm 2,6 \mathrm{mmHg}$ respectivamente; $\mathrm{p}<0,05)$.

\section{Respuesta descriptiva durante el test de ejercicio}

Todos los participantes realizaron el test de Naughton, alcanzando los 5 primeros niveles de intensidad. Entre los niveles 6 y 9 aumentó progresivamente la cantidad de sujetos que abandonó la prueba por intolerancia a la carga de trabajo, llegando sólo 2 niños (varones) al nivel 9. Por otra parte, tanto la FC como el EP aumentaron de manera significativa en cada nivel de intensidad del test de Naughton ( $\mathrm{p}<0,0001)$. No existió diferencia significativa entre géneros en cuanto al EP durante la prueba. Sin embargo, las niñas presentaron una mayor FC que los varones, alcanzando significancia estadística en los niveles 2,4 y $5(\mathrm{p}<0,05)$ (Figura 1A y B). En el máximo nivel de intensidad alcanzado por cada sujeto durante el test, la frecuencia cardíaca media fue

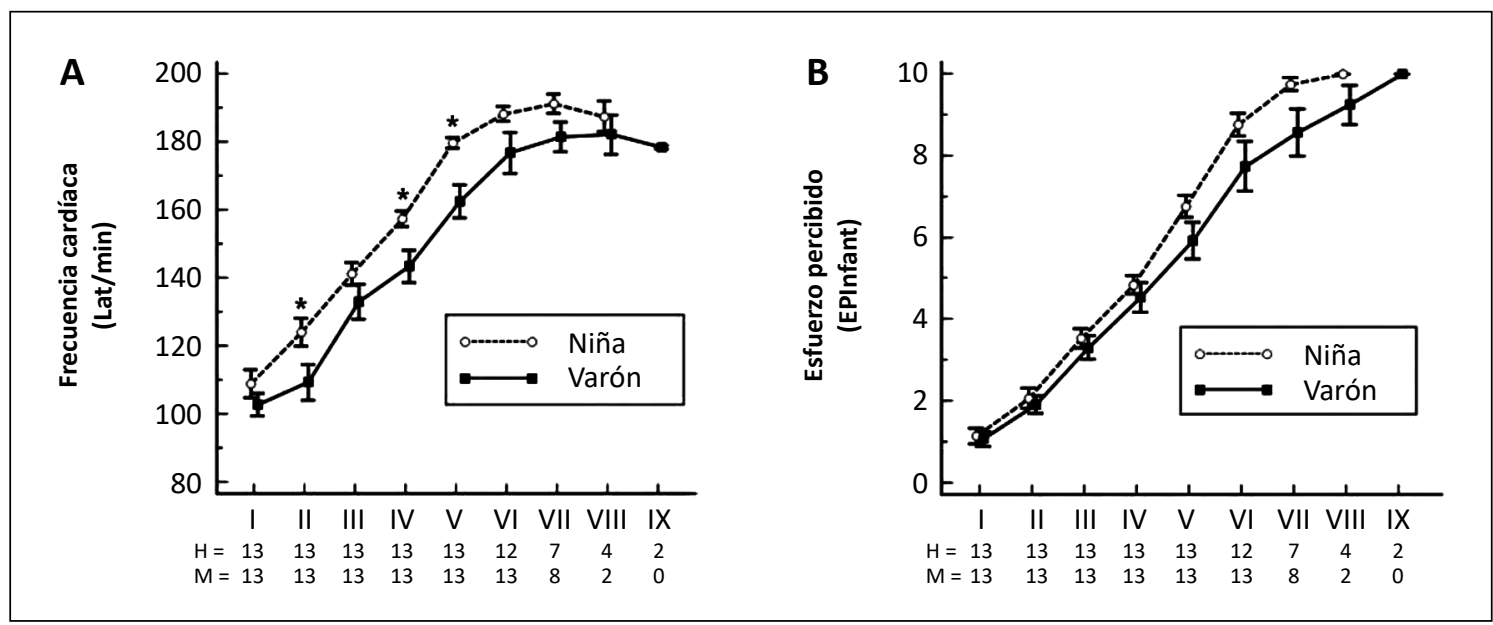

Figura 1. Respuesta fisiológica durante el test de Naughton. En A se muestra la respuesta de la frecuencia cardíaca y en $\mathbf{B}$ la respuesta del esfuerzo percibido. $\mathrm{H}=$ varón; $\mathrm{M}=$ niña. Resultados se muestran en promedio \pm error estándar. $* \mathrm{p}<0,05$ (entre géneros). Los números romanos de la abscisa indican el nivel de intensidad del test de ejercicio. 
Tabla 1. Análisis de regresión lineal de la escala de medición de esfuerzo percibido EPInfant expresado como función de la frecuencia cardíaca (latidos/min), velocidad de carrera e inclinación durante el test de Naughton

\begin{tabular}{|llccccc|}
\hline Variable & Subgrupo & Pendiente & Intercepto & $\mathbf{r}$ & $\mathbf{r}^{\mathbf{2}}$ & Valor de p \\
Frecuencia cardíaca & Todos & 9,6 & 101,6 & 0,89 & 0,80 & $<0,0001$ \\
& Varones & 9,5 & 98,6 & 0,86 & 0,74 & $<0,0001$ \\
& Niñas & 9,5 & 105,5 & 0,94 & 0,89 & $<0,0001$ \\
Velocidad de carrera & Todos & 0,5 & 2,8 & 0,88 & 0,78 & $<0,0001$ \\
& Varones & 0,5 & 2,9 & 0,86 & 0,73 & $<0,0001$ \\
& Niñas & 0,5 & 2,6 & 0,92 & 0,83 & $<0,0001$ \\
\multirow{2}{*}{ Inclinación de la trotadora } & Todos & 1,2 & $-0,3$ & 0,84 & 0,71 & $<0,0001$ \\
& Varones & 1,2 & $-0,03$ & 0,82 & 0,68 & $<0,0001$ \\
& Niñas & 1,2 & $-0,6$ & 0,87 & 0,75 & $<0,0001$ \\
\hline
\end{tabular}

de $182,9 \pm 5,4$ en los varones y $193,3 \pm 1,7$ en las niñas (p: N.S.); y el EP medio fue de $10,0 \pm 0$ en los varones y 9,1 $\pm 0,6$ en las niñas (p: NS).

Validez de criterio de la escala EPInfant: En el análisis de regresión lineal, se determinó que el EP muestra una fuerte correlación con los criterios de referencia, tanto en varones, como niñas. A su vez, no existió diferencia significativa en los coeficientes de correlación entre los subgrupos, como también, entre los criterios de referencia. Adicionalmente, el coeficiente de determinación $r^{2}$ fue $>0,65$ en todos los modelos de regresión; confirmando en esta modalidad de ejercicio, la fuerte interdependencia entre la intensidad de ejercicio y EP (Tabla 1).

\section{Discusión}

Los resultados de este estudio mostraron una fuerte asociación entre el EP y variables de intensidad de ejercicio (FC y carga de trabajo), lo que sugiere que la escala EPInfant tiene una adecuada validez de criterio concurrente para medir la percepción de esfuerzo en niños durante el test de Naughton.

Estos resultados concuerdan con estudios de validación de otras escalas previamente realizados con esta modalidad de ejercicio; en los que se han reportado índices de validez de criterio concurrente aceptables entre el EP y variables de estrés fisiológico, tanto en niños, como en adolescentes. Robertson et al., observaron en niños entre 10 y 14 años niveles de correlación de 0,8 y 0,9 considerando la $\mathrm{FC}$ y $\mathrm{VO}_{2}$ como criterio de referencia ${ }^{9}$. Por su parte, Suminsky et al., en el estudio de validación de la escala OMNI en español, observaron en 68 niños entre 9 y 12 años una fuerte asociación entre el EP y las variables fisiológicas $\mathrm{VO}_{2}$ y $\mathrm{FC}^{10}$. Adicionalmente, Lambrick et al., en niños entre 7 y 8 años, demostraron una significativa interdependencia entre el EP y los parámetros de estrés fisiológico durante una prueba de ejercicio incremental en cinta rodante ${ }^{11}$.

Por otra parte, en nuestro estudio fue observado un alto nivel de correlación entre el EP, tanto con la velocidad $(\mathrm{r}=0,84)$, como con la inclinación de la cinta $(r=0,80)$. En este sentido, podemos destacar que son escasos los estudios que han reportado la asociación entre el EP y la carga de trabajo. Los existentes, se remiten sólo a protocolos experimentales basados en pruebas de ejercicio en ciclo-ergómetro. Y en estos, se han constatado niveles de correlación aceptables entre los watts desarrollados y el EP, lo cual, estaría en concordancia con los hallazgos de nuestro estudio $^{12-14}$.

Todos los coeficientes de la regresión lineal fueron altamente significativos ( $p<0,0001)$, sugiriendo que el EP, medido a través de la escala EPInfant, tendría un alto poder predictivo de estrés fisiológico en esta modalidad de ejercicio. Es importante destacar que en ninguno de los parámetros de referencia considerados existió diferencia absoluta en la magnitud de la pendiente de ascenso entre géneros. Esto permite sostener que el nivel de interdependencia entre el EP y los parámetros de intensidad de ejercicio fue independiente del género en esta modalidad de ejercicio.

En relación a las limitaciones de este estudio es posible mencionar que el estudio fue realizado con una cantidad limitada de sujetos, por lo tanto, no es posible ampliar estas conclusiones a la totalidad de la población blanco. Por otra parte, la totalidad de la muestra completó sólo 5 de los 9 niveles del test de Naughton, lo cual, pudiese afectar la potencia estadística de las asociaciones determinadas; principalmente entre 
los más altos niveles de intensidad del test y las categorías más altas de la escala EPInfant. Pese a lo anterior, un riesgo de error $\alpha$ y $\beta$ inferior al $1 \%$ y $10 \%$ fue calculado en la muestra total. Por otra parte, estudios ulteriores en esta modalidad de ejercicio deben ser dirigidos a determinar la validez de esta escala en sujetos pertenecientes a otras etapas del desarrollo cognitivo, así como también, considerando otros parámetros de referencia como el $\mathrm{VO}_{2}$.

Finamente es posible concluir que la escala EPInfant posee una adecuada validez de criterio concurrente para medir EP durante el test de Naughton en la muestra estudiada; sin embargo, se requiere de más estudios para determinar otras propiedades de medición en esta modalidad de ejercicio.

\section{Agradecimientos}

A cada uno de los participantes por contribuir a la ejecución de este estudio y al establecimiento educacional por el respaldo otorgado en el desarrollo de esta línea de investigación.

\section{Bibliografía}

1.- BORG G. Perceived exertion as an indicator of somatic stress. Scand J Rehabil Med. 1970; 2: 92-8.

2.- BORG G A. Psychophysical bases of perceived exertion. Med Sci Sports Exerc. 1982; 14: 377-81.

3.- GROSLAMBERT A, MAHON A D. Perceived exertion: influence of age and cognitive development. Sports Med 2006; 36: 911-28.

4.- RODRÍGUEZ I, ZAMBRANO L, MANTEROLA C. Criterion-related validity of perceived exertion scales in healty children: a systematic review and meta-analysis. Arch Argent Pediatr 2016; 114: 120-8.

5.- RODRÍGUEZ I, ZENTENO D, CISTERNAS L, RODRÍGUEZ P, REYES G, TRONCOSO K. Construcción y evaluación de EPInfant: una escala para la medición del esfuerzo percibido en población pediátrica. Arch Argent Pediatr 2015; 113: 550-7.

6.- RODRÍGUEZ I. Escala me medición de esfuerzo percibido infantil (EPInfant): validación en niños y adolescentes chilenos. Rev Chil Pediatr 2015; 2016; 87: 211-2.

7.- RODRÍGUEZ I, MANTEROLA C. Validación inicial de la escala de medición de esfuerzo percibido infantil (EPInfant) en niños chilenos. Biomédica 2016; 36: 29 38.

8.- CHEN M J, FAN X, MOE S T. Criterion-related validity of the Borg ratings of perceived exertion scale in healthy individuals: a meta-analysis. J Sports Sci 2002; 20: 873-99.

9.- ROBERTSON R J, GOSS F L, AARON D J, TESSMER K A, GAIROLA A, GHIGIARELLI J J, et al. Observation of perceived exertion in children using the OMNI pictorial scale. Med Sci Sports Exerc 2006; 38: $158-66$

10.- SUMINSKI R R, ROBERTSON R J, GOSS F L, OLVERA N. Validation of the Omni Scale of Perceived Exertion in a sample of Spanish-speaking youth from the USA. Percept Mot Skills 2008; 107: 181-8.

11.- LAMBRICK D M, ROWLANDS A V, ESTON R G. The perceptual response to treadmill exercise using the Eston-Parfitt scale and marble dropping task, in children age 7 to 8 years. Pediatr Exerc Sci 2011; 23: 36-48.

12.- ROBERTSON R J, GOSS F L, BOER N F, PEOPLES $\mathrm{J}$ A, FOREMAN A J, DABAYEBEH I M, et al. Children's OMNI scale of perceived exertion: mixed gender and race validation. Med Sci Sports Exerc 2000; 32: 452-8.

13.- BALASEKARAN G, LOH M K, GOVINDASWAMY V V, ROBERTSON R J. OMNI Scale of Perceived Exertion: mixed gender and race validation for Singapore children during cycle exercise. Eur J Appl Physiol 2012; 112: 3533-46

14.- BALASEKARAN G, LOH M K, GOVINDASWAMY V V, CAI S J. Omni Scale Perceived Exertion responses in obese and normal weight male adolescents during cycle exercise. J Sports Med Phys Fitness 2014; 54 186-96.
Correspondencia a:

Iván Rodríguez Núñez

Laboratorio de Biología del Ejercicio, Facultad de

Ciencias de la Salud, Universidad San Sebastián.

Lientur 1457; edificio Los Maitenes ( $4^{\circ}$ piso).

Concepción, Chile.

Teléfono: 041-2487293.

Email: ivan.rodriguez@uss.cl 